\title{
Reconstruction of the Pulmonary Artery
}

\author{
Federico Venuta, MD, ${ }^{*}$ and Anna Maria Ciccone, $\mathrm{MD}^{\dagger}$
}

\begin{abstract}
Sleeve resection and prosthetic reconstruction of the pulmonary artery have progressively gained acceptance as an alternative to pneumonectomy in lung cancer surgery. Previous concern was mainly related to technical difficulties, intraoperative and postoperative complications, lack of long-term survival, and impact on cardiopulmonary function. For this reason it was not until very recently that lobectomy associated with resection and reconstruction of the pulmonary artery, associated or not to a sleeve resection of the bronchus, has been demonstrated to be an advantageous alternative. The concern about an increased complication rate has been proven to be excessive; in fact, pulmonary artery reconstruction can be performed safely and effectively with the correct indications and technique. We hereby report our experience, along with a review of the indications, the surgical technique, and outcome of pulmonary artery reconstruction.

Semin Thorac Cardiovasc Surg 18:104-108 (c) 2006 Elsevier Inc. All rights reserved.
\end{abstract}

KEYWORDS lung cancer, pulmonary artery reconstruction, bronchial sleeve resection

Sleeve resection and prosthetic reconstruction of the pul$\checkmark$ monary artery (PA) has progressively gained acceptance as an alternative to pneumonectomy in lung cancer surgery. This assumption is now based on solid reports on long-term survival after pulmonary artery reconstruction for lung cancer $^{1-6}$ and on the common opinion that pneumonectomy is a "disease in itself" and should be avoided whenever possible. ${ }^{7}$ Previous concern was mainly related to technical difficulties, intraoperative and postoperative complications, lack of longterm survival, and impact on cardiopulmonary function. ${ }^{8,9}$ The series of 37 PA sleeve resections published by VogtMoykopf in $1986^{10}$ demonstrated that the operation was feasible with acceptable complications and good long-term survival; however, it was not until very recently that lobectomy associated with resection and reconstruction of the PA, associated or not to a sleeve resection of the bronchus, has been demonstrated to be an advantageous alternative to pneumonectomy. The concern about an increased complication rate has been proven to be excessive, and, in our experience, PA reconstruction techniques can be performed safely and effectively with the correct indications and technique.

The pulmonary artery can be infiltrated by primary lung tumors or by metastatic hilar-mediastinal lymph nodes with

*University of Rome "La Sapienza", Department of Thoracic Surgery, Policlinico Umberto I, Rome, Italy

†Ospedale S. Andrea, Rome, Italy.

Address reprint requests to Federico Venuta, MD, Cattedra di Chirurgia Toracica, Policlinico Umberto I, Università di Roma "La Sapienza", V.le del Policlinico, 00100 Rome, Italy. E-mail: sofed@libero.it extracapsular extension; it can be involved to various extents, from partial infiltration to a more extensive and even circumferential invasion. In the first case a simple tangential resection with direct suture is required ${ }^{11}$; this is not conventionally considered as "PA reconstruction" and should be regarded as a simple variation of standard lobectomy. More extensive defects require reconstruction by a patch, by endto-end anastomosis, or by a prosthetic conduit. Invasion of the main PA (T4 lung cancer) requires reconstruction with the use of cardiopulmonary bypass.

Resection and reconstruction of the PA, often associated with sleeve resection of the bronchus, is usually performed to avoid pneumonectomy in patients with lung cancer. This approach is reasonable not only in patients who cannot tolerate pneumonectomy because of impaired cardiopulmonary function, but also in any patient in whom the procedure is feasible, since the oncological value of the procedure has been recently proven (Table 1 ). However, it is often difficult to establish preoperatively the indication for PA reconstruction. Angiography, computed tomography, and magnetic resonance may contribute to assess the extent of infiltration, but the decision is usually made intraoperatively.

The primary indication is direct infiltration of the interlobar PA by the primary tumor; however, extracapsular nodal invasion into the vessel can also be treated effectively by this technique. After induction therapy the PA can be involved not only by residual tumor, but also by desmoplastic reaction, scarring tissue, or fibrosis; also in this subset of patients bronchovascular reconstruction can be performed safely.

Most of the PA reconstructions (more than 70\%) are per- 
Table 1 Reconstruction of the Pulmonary Artery Alone or Associated with a Bronchial Sleeve Resection

\begin{tabular}{|c|c|c|c|c|c|c|}
\hline Author & Year & $\begin{array}{l}\text { No. of } \\
\text { Patients }\end{array}$ & $\begin{array}{l}\text { Complications } \\
\text { (\%) }\end{array}$ & $\begin{array}{l}\text { Technical Complications } \\
\text { (\%) }\end{array}$ & $\begin{array}{l}\text { Mortality } \\
\text { (\%) }\end{array}$ & $\begin{array}{c}5 \text { yr } \\
\text { Survival }\end{array}$ \\
\hline Rendina ${ }^{1}$ & 1999 & 52 & 13.4 & 1.9 & 0 & 38.3 \\
\hline Icard $^{12}$ & 1999 & 16 & NS & NS & NS & $39^{*}$ \\
\hline Lausberg² & 2000 & 4 & NS & 0 & NS & $61.9^{*}$ \\
\hline Okada $^{3}$ & 2000 & 21 & NS & 0 & 0 & $48^{*}$ \\
\hline Fadel $^{13}$ & 2002 & 11 & 12.4 & 1.4 & 0.7 & $52^{*}$ \\
\hline Chunwei ${ }^{4}$ & 2003 & 21 & NS & 0 & NS & $33.3^{*}$ \\
\hline Lausberg $^{5}$ & 2005 & 67 & NS & 0 & 1.5 & $42.9^{*}$ \\
\hline Nagayasu $^{6}$ & 2006 & 29 & 27.6 & NS & 17.2 & $24.2^{*}$ \\
\hline
\end{tabular}

*Overall survival of pulmonary artery and bronchial reconstruction.

formed for left upper lobe tumors; $20 \%$ are performed for right upper lobe lesions and 10\% are performed for tumors involving the main PA or arising in one of the lower lobes.

Preoperative screening does not require special considerations; it should be performed according to the stage of the disease, the clinical status of the patient, and the protocols that are in use at the different centers. In other words it should not be different from the schedule followed for patient candidates to standard lung resections.

\section{Operative Technique}

The operative procedure includes a resection and a reconstruction phase. The first step requires full control of the proximal PA; however, transection of the vessel should be postponed until the feasibility of the procedure is ascertained. The superior pulmonary vein should always be completely dissected and retracted to allow better viewing of the artery, especially on the right side. Once all the elements involved in the resection are fully prepared, $5000 \mathrm{U}$ of heparin sodium are administered and the resection can begin. We usually first transect the vein, both for oncological reasons and to better visualize the artery (upper lobe tumors). The main PA is clamped, as well as the vein corresponding to the residual lobe, to prevent back bleeding at the suture site; this maneuver will also prevent manipulation, compression, and traumatization of the fragile distal stump of the artery.

On the left side, if an upper lobe tumor involves only the interlobar artery, the main PA can be prepared extrapericardially; however, in most cases the artery is involved close to its origin and it must be prepared inside the pericardium. If the tumor infiltrates the artery very proximally, the posterior aspect of the artery is invariably dissected blindly; this maneuver should be carried our very carefully, paying attention not to injure the right main PA, especially when passing the clamp behind the vessel. Once the main PA is fully under control, the fissure is prepared; clamping of the vessel could facilitate the dissection. The artery should be exposed down to the branch for the apical segment of the lower lobe, usually arising posterolaterally, and to the anterior basal segment artery, which continues anteriorly along the curve of the interlobar artery after the lingular branch. Tumors arising in the left lower lobe or infiltrative metastatic lymph nodes located between the lower lobe branches may involve the infe- rior aspect of the interlobar artery. This means that the proximal PA is certainly free from tumor and the preparation is much easier; however, in this situation the viability of the lingular artery should always be ascertained. The main PA can thus be clamped as well as the superior pulmonary vein, and the inferior vein is transacted. The interlobar artery is incised obliquely in an anteroposterior and inferosuperior direction, and the lobe is removed. The defect can be usually reconstructed with a patch that should be adequately trimmed, paying attention to the patency of the lingular artery.

On the right side the anatomy is completely different and it makes proximal tumors often unresectable owing to the invasion of the adjacent structures. PA reconstruction is usually feasible for tumors or lymph nodes located on the anteroinferior aspect of the bronchus and involving the artery between the Boyden branch and the ascending fissural vessel. The proximal control of the artery can be obtained either extrapericardially, retracting the superior vena cava (SVC), or, if required, inside the pericardium. Especially on this side the division of the superior pulmonary vein helps to expose the involved artery; however, this step should be discouraged until the feasibility of the reconstruction is fully ascertained. It is important to demonstrate the integrity of the arteries to the middle lobe and the apical segment of the lower lobe. On this site the neoplastic invasion is usually extended longitudinally, but it does not involve the full circumference of the artery; for this reason a tissue patch is almost always adequate for reconstruction. If the tumor arises in the right lower lobe, the situation is usually similar to its left counterpart.

The techniques of reconstruction, as mentioned before, include different options:

- Patch reconstruction

- Sleeve resection with end-to-end anastomosis

- Sleeve resection and reconstruction by a prosthetic conduit

- Reconstruction of the main PA via cardiopulmonary bypass

\section{Patch Reconstruction After Partial Resection}

This technique allow the repair of the PA in a variety of circumstances: from a limited infiltration to large defects lon- 


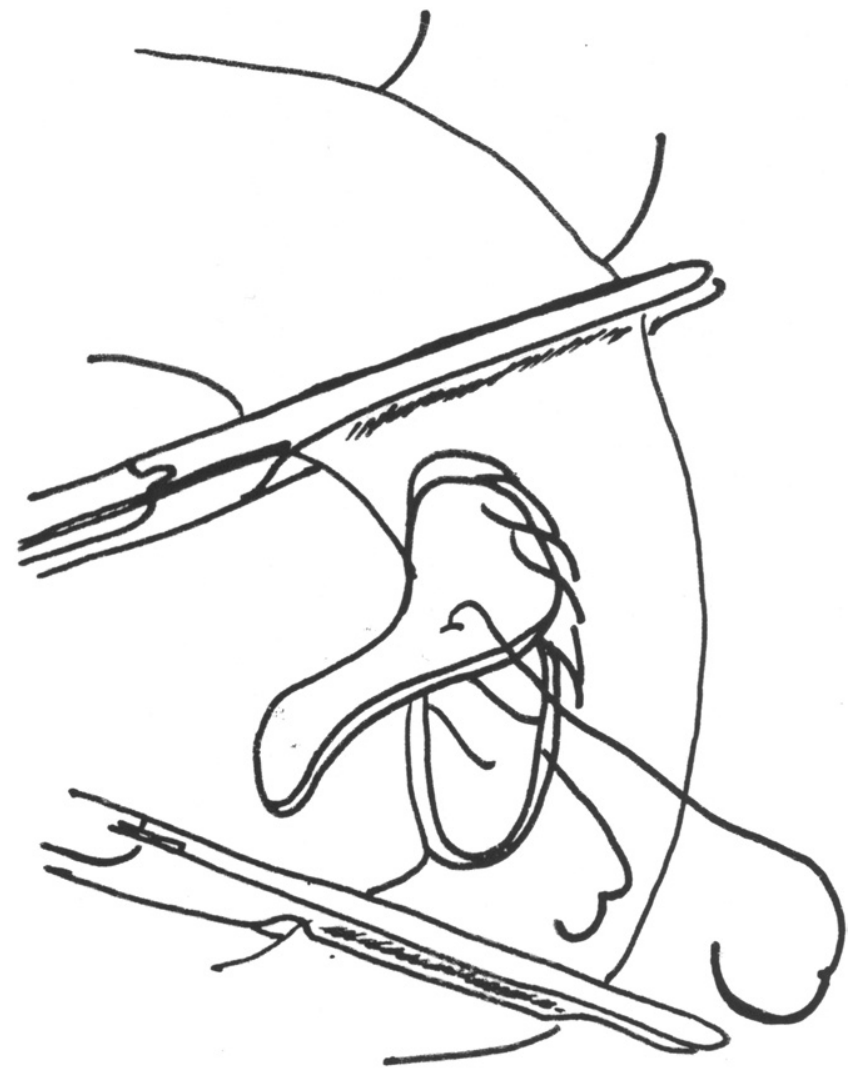

Figure 1 Patch reconstruction of the pulmonary artery.

gitudinally extended on one aspect of the vessel (Fig. 1). Obviously the wall of the artery opposite to the site of the reconstruction should be free from tumor. After resecting the involved artery the defect is usually stretched longitudinally; for this reason the morphology of the patch should be tailored on the resected portion of the vessel. Our choice for reconstruction goes to autologous pericardium, ${ }^{1,14}$ that is, fresh, unpreserved, cost-free, and perfectly biocompatible. It is usually harvested anteriorly to the phrenic nerve, and the defect can be left open. The patch is adequately trimmed. Suturing with a 5-0 monofilament nonabsorbable material must be done very carefully since the edge of the patch tends to shrink and curl and it may create problems of distance between the stitches; stay sutures at the two edges could facilitate suturing. Autologous pericardium may be fixed in diluted glutaraldehyde (two drops of $20 \%$ glutaraldehyde in $50 \mathrm{~mL}$ saline) for 1 minute to let it stiffen and facilitate manipulation during suturing. Other materials have been used, and in particular, bovine pericardium, that helps to overcome pitfalls related to harvesting and trimming, and displays little if any elasticity, showing even and stiff edges.

\section{Reconstruction by End-to-End Anastomosis After Sleeve Resection}

When the vascular and bronchial sleeve resection and reconstruction are performed simultaneously, the bronchial axis is shortened and the two vascular stumps are usually apposed with acceptable tension (Fig. 2). The PA anastomosis should be accomplished after completion of the bronchial anastomosis to reduce manipulation of the vessel; also, exposure and suturing of the two bronchial stumps are improved when the artery is transacted. If the distance between the arterial stumps is deemed excessive, the interposition of a prosthetic conduit is mandatory. The anastomosis is performed by running 5-0 or 6-0 monofilament nonabsorbable material; especially if some degree of tension exists, it could be safer to complete the posterior portion of the suture and subsequently parachute the two stumps together while lifting the lower lobe. Caliber discrepancy between the two sides of the anastomosis is never a problem; the elasticity of the wall of the vessel greatly helps at this stage. However, regular and even margins are desirable even at the cost of some loss of tissue, since it facilitates the correction of large-caliber discrepancy when it occurs.

\section{Reconstruction by a Prosthetic Conduit After Sleeve Resection}

This type of reconstruction is performed in the very unusual case of circumferential defects in which end-to-end anastomosis is not feasible. Interposition of a conduit could be required when a left upper lobe tumor extensively infiltrates the PA, but the corresponding lobar bronchus is not involved (PA sleeve without bronchial sleeve), leaving a long bronchial segment keeping the two vascular edges far away. A tube of autologous pericardium could be designed,${ }^{15}$ trimming it and suturing it over a $28-\mathrm{F}$ chest tube with a 5-0 or 6-0 monofilament of nonabsorbable material. Usually a 2-cm-long conduit is enough to fill the gap between the vascular edges; also in this case the length and size of the pericardial tube should be tailored on the basis of the resected arterial segment. When the blood fills the conduit after flow restoration, the dimension of the conduit will increase by approximately onethird. Lengthening of the PA should be avoided since it may cause kinking, impaired flow, and eventually thrombosis. The use of a PTFE tube has also been reported. ${ }^{16}$

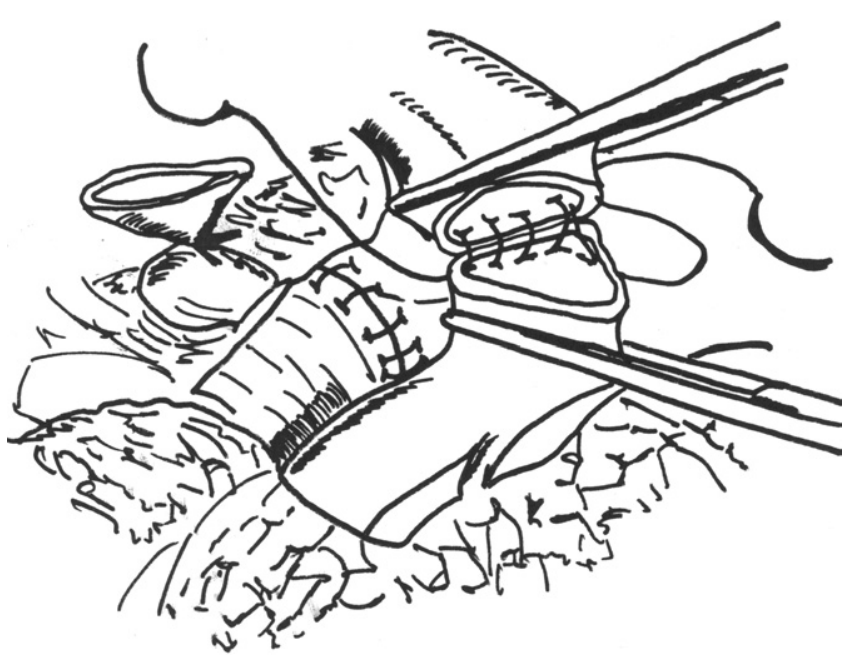

Figure 2 Sleeve resection of the pulmonary artery with end-to-end anastomosis in conjunction with bronchial sleeve resection. 


\section{Reconstruction of the Main Pulmonary Artery via Cardiopulmonary Bypass}

This is a very rare situation caused by proximal invasion of hilar tumors; it is obviously associated with left pneumonectomy. Cardiopulmonary bypass is instituted via median sternotomy. The main PA is separated from the ascending aorta; the right PA is clamped between the ascending aorta and the superior vena cava since usually the tumor does not allow clamping on the left side of the aorta. Alternatively the right heart could be emptied by bicaval cannulation, making PA clamping unnecessary. After resection of the left PA and part of the main PA en bloc with the lung, the defect can be reconstructed by a patch.

\section{Outcome}

The postoperative course after PA reconstruction is strictly related to the choice of the most appropriate procedure for each patient and to operative judgment and technique. Specific complications consist of thrombosis and leakage and can be expected in less than $5 \%$ of the patients. Also bronchoarterial fistula can occur when PA reconstruction is associated with bronchial sleeve lobectomy; however, the interposition of the intercostals muscle between the two structures can contribute to prevent it. ${ }^{17}$ Also kinking and rotation of the reconstructed arterial segment after lung re-expansion may occur if the axis and the length of the artery are not carefully considered during surgery. Stricture of the anastomotic line is extremely rare.

Mid- and long-term evaluation of patients undergoing PA reconstruction is based on the three following issues:

- Patency of the vessel and perfusion of the residual lobe

- Right heart function

- Survival (oncological value of the procedure)

Patency problems usually occur after the operation in less than $2 \%$ of the cases. ${ }^{1}$ In our series only one patient showed multiple bilateral pulmonary embolism, suggesting that the origin of the problem was peripheral. In these cases CT is a versatile noninvasive diagnostic tool. Angio-MR usually provides outstanding imaging and may be useful in demonstrating patency problems also in the immediate postoperative course. Standard angiography still remains an option.

From the cardiological point of view, in the absence of complications like kinking or thrombosis, lobectomy associated with PA reconstruction behaves like standard lobectomy ${ }^{1,5,18}$; previous studies from our group confirmed that right ventricle morphology and function and PA pressure measurements by Doppler echocardiography performed after PA reconstruction equally relates to standard lobectomy ${ }^{1}$; if the residual lobe is normally perfused, the advantages of standard lobectomy over pneumonectomy from the functional point of view have been extensively described in the literature. ${ }^{19-22}$

The reports on PA reconstruction have been rare in the literature so far, even if recently they have been increasing (Table 1). For this reason the long-term survival of these patients has been uncertain. Comparison between the different series is extremely difficult because of the size, demographics, inclusion criteria, and duration of follow-up. However, it has been recently indicated that survival after lobectomy with PA resection and reconstruction is comparable, stage-by-stage, to that reported in the major reviews on lung cancer surgery and sleeve resection ${ }^{2-6,11-13}$ as in our series. ${ }^{1}$ The impact of the nodal status on survival is also comparable with that reported for bronchial sleeve and standard resection. In the face of $\mathrm{N} 1$ or $\mathrm{N} 2$ involvement, once the decision to resect the tumor with intent to cure is taken, PA reconstruction can also be proposed as an adequate procedure in this setting, although some surgeons would still advocate the need of pneumonectomy. Also, there is no statistically significant difference between PA reconstruction alone or PA reconstruction associated with a bronchial sleeve. This suggests that even complex lung-sparing operations can be performed with intent to cure as long as a complete anatomical resection is obtained.

In conclusion, morbidity, mortality, functional results, perfusion scans, and cardiological data of PA reconstruction do not differ from what is currently reported for standard lobectomy. Long-term survival is in line with that reported for standard resection. These data support PA reconstruction as a viable option in the treatment of lung cancer.

\section{References}

1. Rendina EA, De Giacomo T, Venuta F, et al: Sleeve resection and prosthetic reconstruction of the pulmonary artery for lung cancer. Ann Thorac Surg 68:995-1002, 1999

2. Lausberg HF, Graeter TP, Wendler O, et al: Bronchial and bronchovascular sleeve resection for treatment of central lung tumors. Ann Thorac Surg 70:367-372, 2000

3. Okada M, Yamagishi H, Stake S, et al: Survival related to lymph node involvement in lung cancer after sleeve lobectomy compared with pneumonectomy. J Thorac Cardiovasc Surg 119:814-819, 2000

4. Chunwei F, Weiji W, Xinguan Z, et al: Evaluations of bronchoplasty and pulmonary artery reconstruction for bronchogenic carcinoma. Eur J Cardiothorac Surg 23:209-213, 2003

5. Lausberg HF, Graeter TP, Tscholl D, et al: Bronchovascular versus bronchial sleeve resection for central lung tumors. Ann Thorac Surg 79:1147-1152, 2005

6. Nagayasu T, Matsumoto K, Tagawa T, et al: Factors affecting survival after bronchoplasty and broncho-angioplasty for lung cancer: single institutional review of 147 patients. Eur J Cardiothorac Surg 29:585590, 2006

7. Deslauriers J: Discussion of Rendina EA, Venuta F, De Giacomo T, et al. Safety and efficacy of bronchovascular reconstruction after induction chemotherapy for lung cancer. J Thorac Cardiovasc Surg 114:830-837, 1997

8. Gundarsen AE: Segmental resection of the pulmonary artery during left upper lobectomy. J Thorac Cardiovasc Surg 582-586, 1967

9. Kittle CF: Atypical resections of the lung: bronchoplasties, sleeve resections, and segmentectomies - their evolution and present status. Curr Probl Surg 26:57-132, 1989

10. Vogt-Moykopf I, Fritz TH, Meyer G, et al: Bronchoplastic and angioplastic operations in bronchial carcinoma: long term results of a retrospective analysis from 1973 to 1983. Int Surg 71:211-220, 1986

11. Shrager JB, Lambright ES, McGrath CM, et al: Lobectomy with tangential pulmonary artery resection without regard to pulmonary function. Ann Thorac Surg 70(1):234-239, 2000

12. Icard P, Regnard JF, Guibert L, et al: Survival and prognostic factors in patients undergoing parenchymal saving bronchoplastic operation for 
primary lung cancer: a series of 110 consecutive cases. Eur J Cardiothorac Surg 15:426-432, 1999

13. Fadel E, Yildizeli B, Chapelier AR, et al: Sleeve lobectomy for bronchogenic cancers: factors affecting survival. Ann Thorac Surg 74:851-859, 2002

14. Ricci C, Rendina EA, Venuta F, et al: Reconstruction of the pulmonary artery in patients with lung cancer. Ann Thorac Surg 57:627-633, 1994

15. Rendina EA, Venuta F, De Giacomo T, et al: Reconstruction of the pulmonary artery by a conduit of autologous pericardium. J Thorac Cardiovasc Surg 110:867-868, 1995

16. Solli P, Spaggiari L, Grazia F, et al: Double prosthetic replacement of pulmonary artery and superior vena cava and sleeve lobectomy for lung cancer. Eur J Cardiothorac Surg 20:1045-1048, 2001

17. Rendina EA, Venuta F, De Giacomo T, et al: Intercostal pedicle flap in tracheobronchial surgery. Ann Thorac Surg 62:630-631, 1996
18. Deslauriers J, Gaulin P, Beaulieu M, et al: Long term clinical and functional results of sleeve lobectomy for primary lung cancer. J Thorac Cardiovasc Surg 92:871-879, 1996

19. Berend N, Woolcock AJ, Marlin GE, et al: Effects of lobectomy on lung function. Thorax 35:145-150, 1980

20. Pellettier C, Lapointe L, Leblanc P: Effects of lung function on pulmonary function and exercise capacity. Thorax 45:497-502, 1990

21. Mieghem WV, Demedts M: Cardiopulmonary function after lobectomy or pneumonectomy for pulmonary neoplasms. Respir Med 83:199206, 1989

22. Lewis JW Jr, Gabriel F, Bastanfar M, et al: Right ventricular performance in patients undergoing pneumonectomy. Chest 1992;102:63S, 1992 